\title{
Matter-Wave Interferometer for Large Molecules
}

\author{
Björn Brezger, Lucia Hackermüller, Stefan Uttenthaler, Julia Petschinka, Markus Arndt, and Anton Zeilinger* \\ Universität Wien, Institut für Experimentalphysik, Boltzmanngasse 5, A-1090 Wien, Austria
}

(Received 20 November 2001; published 26 February 2002)

\begin{abstract}
We demonstrate a near-field Talbot-Lau interferometer for $\mathrm{C}_{70}$ fullerene molecules. Such interferometers are particularly suitable for larger masses. Using three free-standing gold gratings of $1 \mu \mathrm{m}$ period and a transversally incoherent but velocity-selected molecular beam, we achieve an interference fringe visibility of $40 \%$ with high count rate. Both the high visibility and its velocity dependence are in good agreement with a quantum simulation that takes into account the van der Waals interaction of the molecules with the gratings and are in striking contrast to a classical moiré model.
\end{abstract}

DOI: $10.1103 /$ PhysRevLett.88.100404

Interferometry with matter waves has become a large field of interest throughout the past years [1,2]. It represents a powerful tool for the demonstration of basic quantum phenomena and of matter-wave effects as well as for applications in high precision measurements inertial forces $[3,4]$ and fundamental constants [5].

It is therefore interesting to extend techniques from neutron and atom interferometry to more massive, complex objects, such as large molecules, in order to quantitatively study decoherence by thermal coupling to the environment, to get information about a wide range of molecular properties and to work on novel lithographical or metrological applications [6]. In this Letter, we report the first demonstration of an interferometer for macromolecules, and in particular a Talbot-Lau interferometer for $\mathrm{C}_{70}$. The molecules are in different internal states with many excited vibrational and rotational degrees of freedom. Nevertheless we observe a clear signature for a quantized center-of-mass motion.

Up to now two types of interferometers with molecules have been demonstrated, namely, a Ramsey-Bordé interferometer using $\mathrm{I}_{2}$ [7] and a mechanical Mach-Zehnder interferometer for $\mathrm{Na}_{2}$ [8]. The use of such devices for larger molecules remains, however, a challenge: A direct application of a Ramsey-Bordé interferometer for complex molecules is precluded by the lack of narrow resonant transitions. On the other hand, a simple extrapolation of the Mach-Zehnder interferometer to heavy, fast molecules would require extremely fine gratings, good collimation, or large distances. This is because such an interferometer operates in the Fraunhofer regime, where the characteristic size of a diffraction pattern scales linearly both with the wavelength and with the distance between the diffracting structure and the plane of observation.

A solution to this problem is the Talbot-Lau interferometer, which also consists of three successive gratings but operates in the near-field or Fresnel regime, where the characteristic size of a diffraction pattern scales with the square root of both the wavelength and the distance. A Talbot-Lau interferometer can accept a spatially incoherent beam, which implies that no collimation is needed, and it works with a spatially extended detector. Therefore
PACS numbers: 03.75.Dg, 03.65.Ta, 39.20. +q

Talbot-Lau count rates can exceed those of Mach-Zehnder interferometers by several orders of magnitude.

A Talbot-Lau interferometer has already been demonstrated for potassium atoms [9] and-in the time domain - for sodium atoms in a Bose-Einstein condensate [10]. Because the scaling properties of near-field devices are very favorable in comparison to far-field interferometers, the Talbot-Lau effect has actually been proposed for experiments using quantum objects up to the size of a virus [6,11].

The successful diffraction of fullerenes at both a mechanical [12] and an optical [13] grating has now stimulated a new set of experiments exploring the limits of macromolecule interferometry. The experiment presented here takes advantage of the Talbot effect which is a selfimaging phenomenon that occurs when a periodic structure is illuminated by a coherent beam [14-16]. Images of this grating are then reconstructed at discrete multiples of the Talbot length $L_{T}=d^{2} / \lambda_{\mathrm{dB}}$, where $d$ is the grating constant and $\lambda_{\mathrm{dB}}$ is the de Broglie wave length of the incident object.

Our interferometer consists of three identical gratings which are equally spaced. The first grating acts as a comb of sources which have vanishing mutual coherence. Yet each source is sufficiently small to prepare the required transverse coherence at the second grating. The second grating is then coherently imaged onto the plane of the third grating by the Talbot effect. The images coming from all sources look identical but they are shifted by multiples of the grating constant and therefore overlap in spite of their lack of mutual coherence [16]. The movable third grating blocks or transmits the molecular interference fringes according to its position.

The experimental setup, as sketched in Fig. 1, consists of three free-standing gold gratings (Heidenhain) with a nominal period of $d=(991.25 \pm 0.25) \mathrm{nm}$, an open fraction (ratio of slit width to period) of $f=0.48 \pm 0.02$, a thickness of $b=500 \mathrm{~nm}$, and a huge usable area with a diameter of $16 \mathrm{~mm}$. The distance between the gratings is set to $L_{1}=L_{2}=0.22 \mathrm{~m}$. The whole setup is placed in a vacuum chamber at a pressure of $3 \times 10^{-8}$ mbar. A beam of $\mathrm{C}_{70}$ fullerene molecules is generated by sublimation 


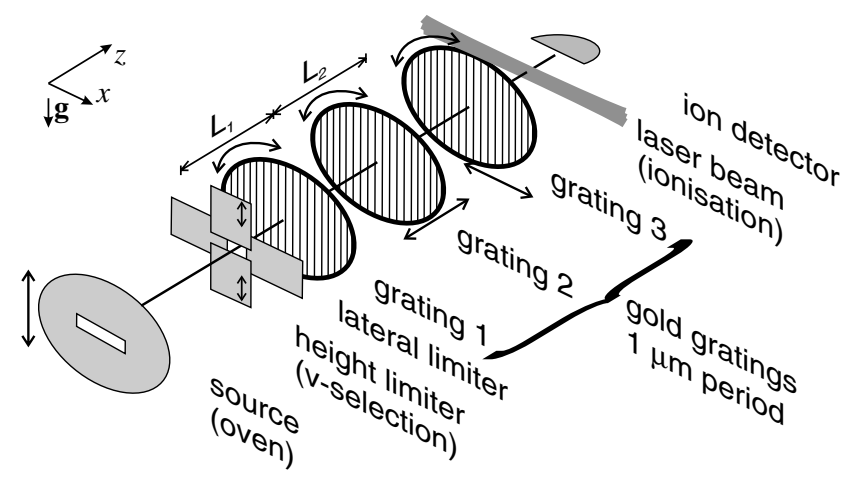

FIG. 1. Setup of the Talbot-Lau interferometer inside the vacuum chamber: It consists of three gratings which are incoherently illuminated by the molecular beam. The interference fringes are detected by transversal scanning of the third grating and integral detection using an ionizing laser beam. The tilt of each grating and the longitudinal position of the second grating are critical and may be adjusted with actuators. The height limiter ensures that only the trajectories within a narrow velocity range, tunable by varying the vertical position of the oven, pass from the oven to the detector.

in an oven at a temperature of $650{ }^{\circ} \mathrm{C}$. Its velocity distribution is close to that of an effusive source, with a most probable velocity of about $200 \mathrm{~m} / \mathrm{s}$. A laser beam with a power of $26 \mathrm{~W}$ traverses the apparatus behind the third grating in the horizontal direction. It is generated by a multiline visible argon ion laser focused to a beam waist of $8 \mu \mathrm{m}\left(1 / e^{2}\right.$ radius $)$. It ionizes the arriving molecules regardless of their transversal position. The interferometer signal is obtained by detecting the resulting ions and scanning the third grating transversally, using an actively stabilized piezotranslation stage (Piezosystem Jena).

The fullerene beam travels $2.38 \mathrm{~m}$ from the oven to the detection laser. In the horizontal direction, the beam is restricted only by the rectangular oven orifice of $1.2 \mathrm{~mm}$ length and a $500 \mu \mathrm{m}$ wide slit that determines the used segment of the first grating. This means that in comparison to the diffraction angles of about $1 \mu \mathrm{rad}$, the illumination is transversally incoherent.

In the vertical direction, the oven orifice of $200 \mu \mathrm{m}$ height and the laser beam fix two regions where the trajectories of the detected molecules are tightly confined in comparison to a typical free-fall distance during their flight. Halfway in between, the vertical positions of the detected molecules are therefore correlated with their longitudinal velocities. We introduce an adjustable slit (Piezosystem Jena) that limits the beam height to $150 \mu \mathrm{m}$ or less at a distance of $1.38 \mathrm{~m}$ from the oven, $0.12 \mathrm{~m}$ before the first grating. It selects a narrow velocity distribution, which has been measured using a mechanical chopper in front of the oven orifice. Its center may be varied between 80 and $215 \mathrm{~m} / \mathrm{s}$ by adjusting the vertical oven position. This corresponds to de Broglie wavelengths of 5.9 and $2.2 \mathrm{pm}$, respectively. The FWHM of the wavelength distribution goes up from $8 \%$ of the mean to $35 \%$ with decreasing center wavelength.
Figure 2 shows the detected signal from a single scan at nearly optimal settings for maximum contrast. Apart from some noise it corresponds well to a sine, as expected from theory for our open fractions near 0.5 , which suppress higher Fourier components. Fast-Fourier transformation (FFT) gives a sharp single peak corresponding to the lattice period. A signal-to-noise ratio of 50:1 was achieved in just $150 \mathrm{~s}$ of total measuring time. It was not necessary to correct the extracted visibilities for dark counts because the dark count rate was about $0.2 \mathrm{~s}^{-1}$ compared to count rates in the range from 50 to $450 \mathrm{~s}^{-1}$ at central velocities of 80 and $160 \mathrm{~m} / \mathrm{s}$, respectively [17]. The phase of the peak FFT component gives the spatial position of the fringe pattern. Comparison of subsequent scans gives the lateral drift of the three-grating setup, which is of the order of $2 \mathrm{~nm} / \mathrm{min}$.

Tilting the three gratings with respect to each other diminishes the visibility. Each grating was prealigned to several milliradians with respect to gravity by observing a laser diffraction pattern outside the vacuum chamber. Fine adjustment of the grating parallelism-better than $2 \mathrm{mrad}$ - was achieved by maximizing the fullerene fringe visibility. A much smaller variation of the visibility was observed when all three gratings were tilted by the same amount. This is because the calculated interferometer phase shift from gravitation is $\Delta \varphi_{g}=0.2 \mathrm{rad}$ per mrad of tilt (see below) and only its nonuniformity due to the finite velocity distribution diminishes the visibility.

Longitudinal displacement of one grating also affects the visibility. Again, the requirement $L_{1}=L_{2}$ was prealigned before evacuating the chamber and fine-aligned on the $100 \mu \mathrm{m}$ level by maximizing the contrast. Vibration isolation of the optical table by pneumatic feet proved to be crucial for obtaining high visibility.

The appearance of a periodic signal as a function of the position of the third grating is not necessarily a sign of quantum interference - it could also result from classical moiré fringes, i.e., shadow patterns which result from the

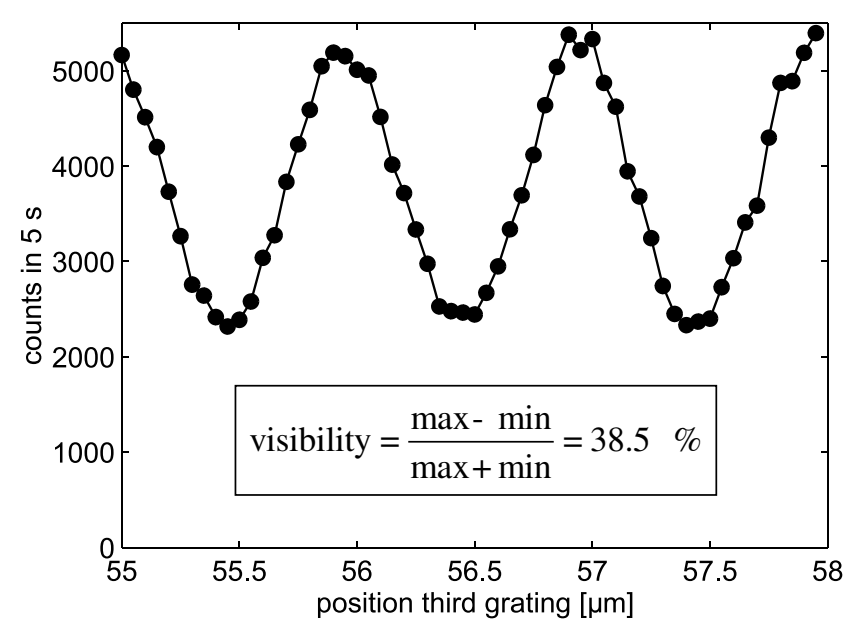

FIG. 2. Interference fringes (raw data) resulting from a typical single scan of the third grating. A central velocity of $115 \mathrm{~m} / \mathrm{s}$ was selected. 
geometry of straight rays passing the gratings or being blocked at one of them [18]. These patterns do not depend on the particle velocity, in sharp contrast to the behavior of quantum de Broglie waves: Here the velocity gives the wavelength, hence the Talbot length, and the ratio $L_{1} / L_{T}$ of the grating separation to the Talbot length is a crucial parameter in the theory of wave propagation.

The quantitative evaluation of the expected velocity dependence involved numerical simulations of the threegrating setup in a quantum de Broglie wave model, in comparison with a classical point-particle calculation. The quantum model relies on Fresnel integrals and calculates them efficiently by a Fourier transform method under the assumption of incoherent illumination. Assuming binary transmission functions corresponding to our grating geometry, the classical point-particle model yields a velocity-independent fringe visibility of $5 \%$, whereas the quantum model gives the dashed curve in Fig. 3 with two maxima slightly below $30 \%$ in the experimentally accessible velocity range. A relative minimum in between corresponds to the velocity $v_{T}=107 \mathrm{~m} / \mathrm{s}$ where $L_{T}=L_{1}$. In this simulation and the following ones, our measured velocity distributions have been taken into account.

The experimental visibility curve (diamonds in Fig. 3) exhibits a pronounced velocity dependence and a maximum visibility of $35 \%$ in the vicinity of the oneTalbot-length criterion. On other days, up to $39 \%$ was obtained with the same settings, and $41 \%$ with a narrower height limiter and therefore a narrower velocity distribution. This is totally incompatible with classical moiré fringes but corresponds also quite poorly to the described quantum simulation result.

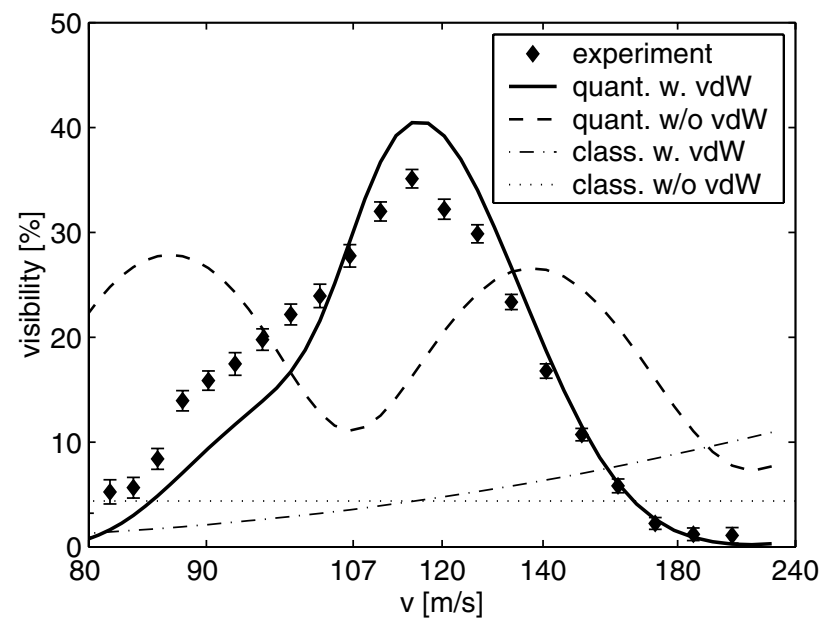

FIG. 3. Dependence of the interference fringe visibility on the mean velocity of the molecular beam. Numerical simulation results are plotted for four models without free parameters: classical or quantum behavior, with or without consideration of the van der Waals (vdW) interaction of the molecules with the second grating. The quantum result including the van der Waals effect is clearly the only adequate one.
The most likely reason for this discrepancy is that the ansatz of a purely absorptive grating transmission function is not adequate: The outcome of far-field diffraction experiments with rare-gas atoms [19] is described correctly only by a theory that includes the phase shift by the van der Waals interaction between the particles and the grating. To estimate the magnitude of this effect for our experimental situation, we assumed a potential $V(r)=-C_{3} r^{-3}$ for a molecule at a distance $r \gtrsim 1 \mathrm{~nm}$ from a gold surface. The constant $C_{3}$ is known for various noble gases [20] near gold surfaces and depends roughly linearly on their atomic dc polarizability. We extrapolated to the known dc polarizability $\alpha_{\mathrm{dc}}=97 \AA^{3} \times 4 \pi \epsilon_{0}$ of the fullerene molecule $\mathrm{C}_{70}$ [21] and obtained the rough estimate $C_{3}^{\left(\mathrm{Au}-\mathrm{C}_{70}\right)} \approx 0.09 \mathrm{eV} \mathrm{nm}^{3}$. For the passage through a grating slit of width $f d(=475 \mathrm{~nm})$ centered at $x=0$, we obtain

$$
\begin{aligned}
& \int V[x, z(t)] d t \approx-\frac{b}{v_{z}} C_{3}[(f d / 2-x)^{-3} \\
&\left.+(f d / 2+x)^{-3}\right] .
\end{aligned}
$$

In the quantum model the potential of Eq. (1) leads to a phase variation of the de Broglie waves passing at different positions $x$ through an individual slit of the second grating. In the classical model we expect the potential gradient to lead to a position-dependent force which ultimately makes an individual slit appear as a minilens with a velocity-dependent focal length. By a simple geometrical argument, one expects maximal visibility for $f_{v} \approx-L_{1}$, for our parameters at velocities about $1000 \mathrm{~m} / \mathrm{s}$. Indeed, our classical simulation shows a single broad maximum of $18 \%$ height in this range. In the experimentally accessible velocity range, it predicts much lower visibilities than our experimental data (dash-dotted line in Fig. 3). On the other hand, the measured functional dependence of fringe visibility on velocity corresponds reasonably well to the numerical quantum model with van der Waals interaction included (solid line in Fig. 3): The position of the single maximum coincides perfectly, and the maximum experimental contrast attains nearly what is expected from theory. Both the theoretical and the experimental curves show a single maximum at $115 \mathrm{~m} / \mathrm{s}$ and are therefore asymmetrical with respect to the criterion $L_{1}=L_{T}-$ a consequence of the complex transmission function of the combined absorptive and phase grating. The remaining discrepancy is not astonishing, having in mind the rather coarse approximations underlying Eq. (1), such as the neglect of edge effects, and the inevitable experimental imperfections.

Talbot-Lau interferometers allow various interferometric measurements, especially those of inertial forces or of decoherence effects, which do not require spatially separated beams. As a first example, we have investigated the gravitational phase shift in our interferometer by tilting the optical table with the whole experimental setup by a few milliradians. As expected [18], the interference fringes are shifted by a phase of $\Delta \varphi_{g}=2 \pi L_{1}^{2} g \alpha /\left(d v^{2}\right)=0.2 \mathrm{rad}$ 
per mrad of table inclination $\alpha$. This means that the gravitational acceleration component in the interferometer plane, which is on the order of $10^{-3} \mathrm{~g}$, can be measured within a short integration time.

In conclusion, we have demonstrated for the first time an interferometer for massive molecules that are internally in a highly excited thermal state. The fact that the experimental visibility is not significantly lower than the theoretical expectation shows that decoherence by emission of blackbody radiation does not play a significant role in this experiment. However, there is a chance to study decoherence in future experiments involving laser heating of the fullerene molecules.

The successful implementation of this near-field interferometer paves the way towards interference of even more massive objects. Talbot-Lau interferometry has a favorable scaling behavior. If one leaves the longitudinal length scale $L_{T}=d^{2} / \lambda$ unchanged, a reduction of the grating period by a factor of 4 already allows the observation of quantum interference with 16 times shorter de Broglie wavelength - that means 16 times heavier molecules, if one assumes a beam of equal velocity, or even 256 times with effusive characteristics at the same temperature. However, the present experiment teaches us that one has to take into account the effect of the van der Waals interaction. With a decreasing grating period the influence of the $r^{-3}$ potential increases strongly. In numerical simulations, the resulting contributions of high diffraction orders at the second grating restrict the self-imaging phenomenon and therefore the fringe visibility to extremely narrow velocity ranges, e.g., $\Delta v / v \approx 0.01$ for $m=16 m_{\mathrm{C}_{70}}$ and $d=d_{\mathrm{C}_{70}} / 4$, assuming both an unchanged velocity and an unchanged $C_{3}$ constant for simplicity. For experimentally accessible velocity distributions, the inclusion of the van der Waals interaction gives therefore a more stringent limit to interferometer scalability than previously discussed by Schmiedmayer et al. [2].

For an experimental implementation, it seems therefore more promising to implement the second grating as an optical phase grating like the one we recently demonstrated for fullerenes [13]. Exploiting the position-dependent ionization and fragmentation probability of molecules in a strong standing light wave, one could also replace the first and third gratings by laser light, thereby eliminating the fragile free-standing microstructures [22].

We acknowledge help in the design of the experiment by Gerbrand van der Zouw and William Case. This work has been supported by the European TMR network, Contract No. ERBFMRXCT960002, by the Austrian Science Foundation (FWF), within the projects F1505 and START
Y177, and by the Marie Curie Fellowship HPMF-CT2000-00797 of the European Community (B. B.).

*Email address: zeilinger-office@exp.univie.ac.at www.quantum.univie.ac.at

[1] Special issue edited by A. Aspect and J. D. Dalibard, C.R. Acad. Sci. Ser. IV 2, No. 4 (2001).

[2] Atom Interferometry, edited by P. R. Berman (Academic Press, San Diego, 1997).

[3] H. Rauch and S. A. Werner, Neutron Interferometry (Oxford University Press, New York, 2000).

[4] T. L. Gustavson, P. Bouyer, and M. A. Kasevich, Phys. Rev. Lett. 78, 2046 (1997).

[5] D. S. Weiss, B. C. Young, and S. Chu, Phys. Rev. Lett. 70, 2706 (1993).

[6] M. Arndt, O. Nairz, and A. Zeilinger, in Quantum [Un]Speakables, edited by R. Bertlmann and A. Zeilinger (Springer, New York, 2002).

[7] C. Bordé, N. Courtier, F. D. Burck, A. Goncharov, and M. Gorlicki, Phys. Lett. A 188, 187 (1994).

[8] M.S. Chapman, C. R. Ekstrom, T. D. Hammond, R. A. Rubenstein, J. Schmiedmayer, S. Wehinger, and D.E. Pritchard, Phys. Rev. Lett. 74, 4783 (1995).

[9] J. F. Clauser and S. Li, Phys. Rev. A 49, R2213 (1994).

[10] L. Deng, E. W. Hagley, J. Denschlag, J. E. Simsarian, M. Edwards, C. W. Clark, K. Helmerson, S. L. Rolston, and W. D. Phillips, Phys. Rev. Lett. 83, 5407 (1999).

[11] J. Clauser, in Experimental Metaphysics, edited by R. Cohen et al. (Kluwer, Dordrecht, 1997).

[12] M. Arndt, O. Nairz, J. Voss-Andreae, C. Keller, G. V. der Zouw, and A. Zeilinger, Nature (London) 401, 680 (1999).

[13] O. Nairz, B. Brezger, M. Arndt, and A. Zeilinger, Phys. Rev. Lett. 87, 160401 (2001).

[14] H. F. Talbot, Philos. Mag. 9, 401 (1836).

[15] M.S. Chapman, C.R. Ekstrom, T. D. Hammond, J. Schmiedmayer, B.E. Tannian, S. Wehinger, and D. E. Pritchard, Phys. Rev. A 51, R14 (1995).

[16] K. Patorski, Prog. Opt. 27, 1 (1989).

[17] These specific values refer to Fig. 3.

[18] M. K. Oberthaler, S. Bernet, E. M. Rasel, J. Schmiedmayer, and A. Zeilinger, Phys. Rev. A 54, 3165 (1996).

[19] R.E. Grisenti, W. Schöllkopf, J.P. Toennies, G. C. Hegerfeldt, and T. Köhler, Phys. Rev. Lett. 83, 1755 (1999).

[20] G. Vidali, G. Ihm, H. Y. Kim, and M. W. Cole, Surf. Sci. Rep. 12, 133 (1991).

[21] M.S. Dresselhaus, G. Dresselhaus, and P.C. Eklund, Science of Fullerenes and Carbon Nanotubes (Academic Press, San Diego, 1998), 2nd ed.

[22] Combinations of absorptive and phase gratings have been discussed for the quite different case of atoms by B. Dubetsky and P. R. Berman [Phys. Rev. A 59, 2269 (1999)]. 